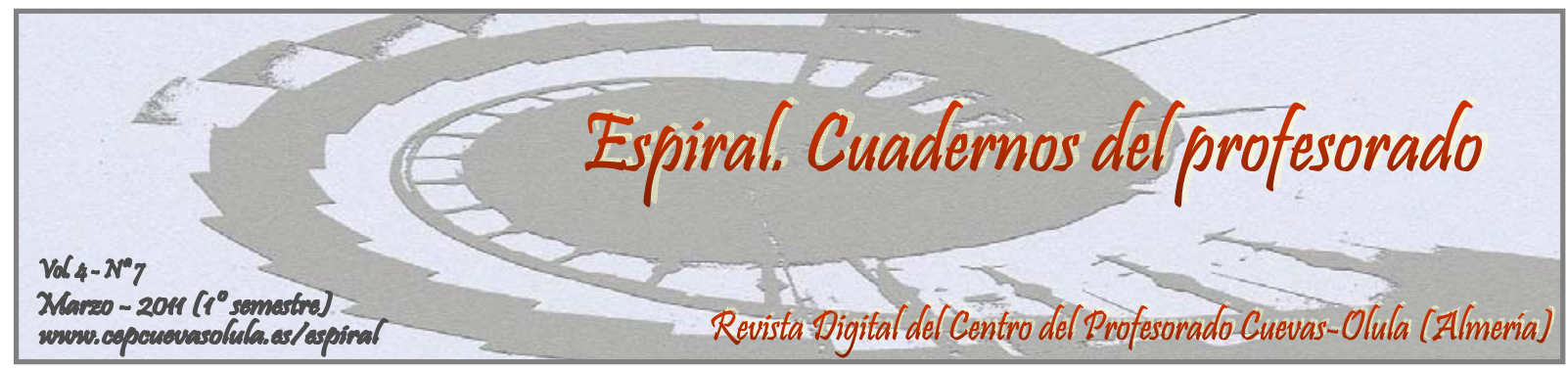

\title{
LA EDUCACIÓN INTERCULTURAL EN UNA ENCRUCIJADA DE CAMINOS: REFLEXIONES PEDAGÓGICAS PARA LA CONSTRUCCIÓN DE UNA ESCUELA INTERCULTURAL
}

\author{
INTERCULTURAL EDUCATION IN A CROSSROADS: PEDAGOGICAL \\ REFLECTIONS ON THE CONSTRUCTION OF AN INTERCULTURAL SCHOOL
}

\author{
Juan J. Leiva Olivencia
}

Departamento de Didáctica y Organización Escolar, Universidad de Málaga, España

\begin{abstract}
RESUMEN: Educar para la diversidad cultural y en la interculturalidad supone una actitud de valoración positiva hacia la comunicación e interacción entre culturas, y hacia la comprensión de lo diverso como un factor de aprendizaje positivo y necesario en las actuales organizaciones escolares. Las actuales escuelas serán inclusivas e interculturales en la medida en que acepten y se enriquezcan con la interculturalidad como construcción educativa cooperativa dirigida fundamentalmente a mejorar la convivencia escolar. En este trabajo se plantea que la interculturalidad se configura ya hoy como un elemento prioritario en el pensamiento pedagógico teórico y práctico de los docentes. Ahora bien, el reto es complejo, y en la actualidad la educación intercultural se encuentra entre el deseo de ser una propuesta educativa crítica, y la realidad de encontrarse en una encrucijada de caminos que van desde la exaltación folclórica de las culturas del alumnado de origen inmigrante al enfoque curricular compensatorio. El objetivo es suscitar el debate y la reflexión pedagógica sobre los caminos prácticos que se vienen trazando en la educación intercultural y que permita la generación de una escuela inclusiva e intercultural.
\end{abstract}

Palabras clave: educación intercultural, profesorado, escuelas inclusivas.

ABSTRACT: Educating for cultural diversity and multiculturalism is a positive assessment attitude towards communication and interaction between cultures, and to the understanding of diversity as a positive and necessary learning factor in the current school organizations. Existing schools will be inclusive and intercultural so far as to accept and be enriched by multiculturalism as cooperative educational building primarily aimed at improving school life. This paper argues that interculturality today is already configured as a priority in the theoretical and practical pedagogical thinking of teachers. Now the challenge is complex, and intercultural education today is between the desire to be a critical educational approach, and the reality of being at a crossroads exaltation ranging from folk cultures of origin of students compensatory immigrant curricular approach. Our objective is to encourage debate and reflection on teaching practical ways that have been drawing on intercultural education and to allow the generation of an inclusive and intercultural school.

Key words: intercultural education, teachers, inclusive schools. 
Leiva Olivencia, J.J. (2011). La educación intercultural en una encrucijada de caminos: reflexiones pedagógicas para la construcción de una escuela intercultural. Espiral. Cuadernos del Profesorado [en línea], 4(7), 43-56. Disponible en: http://www.cepcuevasolula.es/espiral.

Fecha de recepción: 09/09/2010

Fecha de aceptación: 08/01/2011
Enviar correspondencia a: juanleiva@uma.es
1.- LA DIVERSIDAD CULTURAL Y LA INTERCULTURALIDAD EN LA ESCUELA.

En nuestro país, y según los últimos datos ofrecidos por el Ministerio de Educación, y recogidos en el Informe Anual "Datos y Cifras. Curso escolar 2009/2010” (MEC, 2009), el número de alumnos de origen inmigrante pasó de 702.392 en el curso 2007-2008 a 743.696 en el 2008-2009, representando casi un $10 \%$ de la población total de alumnado escolarizado en las enseñanzas de régimen general no universitarias en este último curso. En Andalucía, Comunidad Autónoma donde el fenómeno de la inmigración ha sido muy significativo en los últimos años los datos son ciertamente relevantes y esclarecedores. Según los datos publicados por la Unidad Estadística de la Consejería de Educación de la Junta de Andalucía en Mayo de 2010, en el curso 2009/2010, los centros educativos andaluces acogían ya a un total de 101.838 escolares inmigrantes (CEJA, 2010). Concretamente, en el caso de la provincia de Málaga, sus centros acogían ya a 30.084 alumnos inmigrantes, casi el doble de escasamente hace cuatro cursos académicos, cuando acogía a 16.994 (curso 2005/2006). Estos datos hablan por sí solos, de la emergencia y pujanza de la inmigración y, por tanto, de toda esa diversidad cultural que está transformando el panorama educativo de nuestras instituciones escolares. Hoy en día, nadie pone en duda que la atención a la diversidad cultural supone un reto de primer orden para fortalecer la equidad y la igualdad en nuestro sistema educativo. Su importancia la entendemos en el esfuerzo que toda la comunidad educativa viene desarrollando por garantizar la promoción positiva de la diversidad cultural como una garantía de cohesión social, de solidaridad y, por tanto, una respuesta a la necesidad de mejorar la convivencia educativa y también social (Santos Rego, 2009).

Verdaderamente nuestras escuelas están afrontando el reto de la convivencia intercultural, y no cabe lugar a dudas cuando afirmamos que aulas y escuelas hayan pasado en poco tiempo de ser espacios monoculturales a multiculturales, y con la intención de aspirar a construir de manera cooperativa una educación intercultural que ahonde en la inclusividad escolar. Educar para la convivencia intercultural es la propuesta de acción pedagógica de carácter inclusivo que atiende a esa necesidad, la de responder a formar en el respeto de la diversidad cultural a todos los niños, jóvenes y mayores en una sociedad cada vez más heterogénea y plural. Según Aguado (2003), la educación intercultural nutre e impregna los principios de una educación inclusiva, donde el referente pedagógico por excelencia es la vivencia y convivencia de la diferencia cultural y social como factor de enriquecimiento educativo. Aprovechar educativamente la diversidad cultural pasa por reconocer que lo común es la diversidad y la diversidad debe ser, desde la perspectiva pedagógica, lo común en los diseños y propuestas de intervención educativa que se consideren, ya no sólo oportunos y adecuados, sino imprescindibles en una sociedad democrática como la nuestra (López Melero, 2004). Una sociedad que afronte el trascendental reto de dar respuesta educativa a la diversidad cultural, y sobre todo, que necesita de propuestas pedagógicas que hagan posible la premisa básica de aprender a vivir juntos.

Para Jordán (2007), los sistemas educativos deben asegurar unas bases sólidas para la igualdad de oportunidades en la escuela, el trabajo y la sociedad, atendiendo a la diversidad de géneros, etnias y culturas a partir de un reconocimiento entre iguales basado en el respeto a la diversidad. Así pues, la educación intercultural, entendida como actitud pedagógica que favorece la interacción entre las diferentes culturas, se convierte así en la mejor forma de prevenir el racismo y la xenofobia, así como de promover una convivencia basada en el respeto a la diferencia cultural como legítima en su diferencia. Decir esto no significa que obviemos los conflictos que pueden acontecer en los contextos en los que la diversidad cultural tiene 
una mayor presencia. No obstante, defendemos la idea de que los conflictos denominados interculturales tienen un menor peso en la realidad escolar de lo que los medios de comunicación pretender transmitir, o incluso de las ideas preconcebidas que en un momento dado podemos tener (Leiva, 2010). Es más, son muchos los docentes quienes consideran que los conflictos escolares tienen una vertiente más social que cultural, y que los conflictos que se desarrollan en contextos educativos caracterizados por una significativa diversidad cultural hunden sus raíces en problemáticas sociales y familiares más que en cuestiones identitarias o culturales.

En todo caso, cabe señalar que el nuevo marco normativo que establece la Ley Orgánica 2/2006, de 3 de mayo, de Educación (LOE) define a la escuela pública como un espacio de convivencia y aprendizaje, que ofrece un servicio que garantiza el derecho a la educación de todos los ciudadanos y ciudadanas, y que atienda a la diversidad cultural como un elemento educativo de primer orden. La LOE, en sus fines y principios, opta por un modelo de persona y de sociedad que se fundamenta en un conjunto de valores -justicia, tolerancia, libertad, paz, cooperación, solidaridad, no discriminación, etc.y en los principios democráticos de convivencia: pluralismo, participación y respeto. Así, podemos compartir la idea de que nos encontramos ante un marco normativo favorecedor del desarrollo práctico de la educación intercultural en la escuela, no sólo porque implica el respeto y aprovechamiento de la diversidad cultural, sino que además posibilita el planteamiento de focalizar una cultura de la diversidad en la escuela que significa ir más allá de la interculturalidad en términos de conocimiento cultural. Es decir, la educación intercultural es la propuesta educativa inclusiva que favorece el conocimiento y el intercambio cultural, así como la valoración positiva de la diversidad cultural en términos de convivencia, innovación curricular y fomento de la participación comunitaria en una escuela inclusiva, una escuela para todas y todos, donde alumnado, profesorado, familias y agentes sociales conformen una comunidad educativa abierta.

En efecto, la interculturalidad en la escuela es un paso educativo de relevancia social en la medida en que se considera que ayuda y favorece la constitución de una cultura de la diversidad que va más allá de aceptar sin más la diversidad cultural en una escuela, un barrio o una ciudad. Estamos haciendo referencia a que la educación intercultural es un vehículo de apertura y de fomento de la formación para la participación de todos los miembros de la comunidad educativa (Banks, 2008). Esto supone que cualquier acción o medida educativa denominada intercultural y concebida en el contexto escolar, puede y tiene la potencialidad de influir y generar procesos educativos de carácter comunitario. Es decir, la educación intercultural en la escuela es el primer paso de intervenciones educativas interculturales que no deben restringirse al ámbito puramente académico, aunque como es lógico pensar es obvio que la escuela es un escenario privilegiado para el desarrollo práctico de la interculturalidad. Es más, la institución escolar se convierte así en un lugar fundamental de formación para la convivencia intercultural, por ser un contexto ideal para que alumnos y adultos (profesorado y familias) concreten y se ejerciten en los valores democráticos y de la diversidad. Desde este enfoque, la escuela intercultural es inclusiva y viceversa, ya que debe ser una institución donde hombres y mujeres aprendan valores y actitudes que aseguran una convivencia en la diversidad personal y cultural libre, pacífica, respetuosa y no discriminatoria.

\section{2.- EL PROFESORADO ANTE EL DESAFÍO DE LA INTERCULTURALIDAD: ENFOQUES EDUCATIVOS.}

El profesorado es el agente clave para la construcción de una escuela inclusiva de calidad, ya que es el instrumento pedagógico por excelencia (Jordán, 2007). En el caso de la construcción de una escuela intercultural, cuya calidad esté definida precisamente por la perspectiva intercultural de la propia diversidad cultural de su alumnado y de sus familias, su importancia es si cabe mayor, pues el reto actual de la diversidad cultural, fenómeno que está configurando un nuevo escenario educativo en nuestras aulas y escuelas, es cada vez más emergente e ineludible. Hasta hace escasamente una década, pocos eran los profesores que tenían en mente como un elemento prioritario de la educación la diversidad cultural.

En este sentido, el docente, como profesional comprometido con el análisis de los cambios sociales, viene observando en los últimos tiempos cómo sus aulas y escuelas están pasando de ser espacios culturales más o menos homogéneos a nítidamente plurales y 
heterogéneos, lo cual ha suscitado importantes inquietudes e interrogantes pedagógicas y didácticas entre los profesores. $\mathrm{La}$ multiculturalidad que define la situación escolar de muchos centros escolares viene dada por la existencia cierta de alumnos procedentes de diferentes lugares $\mathrm{y}$ culturas en espacios educativos comunes; algo, que en ningún momento constituye un esfuerzo educativo intercultural, sino que en verdad es una situación dada, real y objetiva. No obstante, ese cambio cualitativo -y también cuantitativo- ha tenido grandes repercusiones para el profesorado de nuestro país. En pocos años, la presencia creciente de alumnos de origen inmigrante ha supuesto todo un cambio en la fisonomía de nuestras aulas y escuelas, lo que ha supuesto la necesaria respuesta desde los principios de una educación democrática en una sociedad plural como la nuestra.

No es nuevo afirmar que el papel del profesorado en esta nueva configuración de la escuela es absolutamente trascendental. Ningún equipo directivo ni ningún docente bien formado y con ilusión por trabajar más y mejor obvia la necesidad de educar en y para la interculturalidad en su centro educativo (Essomba, 2006). Esto se hace más inexcusable cuando los docentes desarrollan su labor formativa en contextos educativos donde la diversidad cultural es lo común y lo característico. Es más, el papel de los docentes como educadores y no como meros transmisores de información (y de cultura) escolar monolítica ha cambiado profundamente y ha supuesto unas nuevas responsabilidades sociales en el campo de la educación, puesto que han asumido el deber de fomentar en la escuela un espíritu tolerante, de respeto y convivencia en el marco de los principios democráticos de igualdad de oportunidades y de respecto en la construcción identitaria.

Sin embargo, hemos de admitir, que en este escenario claramente multicultural, de diversidad cultural del alumnado en las escuelas actuales, hay profesores que todavía permanecen pasivos aunque cada vez son menos-, lo cual tiene significativas repercusiones en el funcionamiento de una escuela democrática, plural y heterogénea (Bartolomé, 2002). En efecto, muchas actitudes de inhibición o de pasividad ante esta realidad de diversidad cultural es una clave negativa si las escuelas quieren convertirse en espacios donde el aprender a convivir en la diferencia cultural sea una clave fundamental de buenas prácticas docentes. En este punto, cabe destacar la importancia de diferentes estudios que se han desarrollado en nuestro país sobre la perspectiva de los docentes ante la interculturalidad (Bartolomé, 2002; Essomba, 2006; Jordán, 1999 y 2007; Leiva, 2008 y 2010; Montón, 2004; Soriano, 2008), tanto a partir de investigaciones realizadas en contextos educativos de diversas regiones y provincias españolas, como de revisiones teóricas de estudios desarrollados en países de nuestro entorno europeo. Estas investigaciones han estudiado principalmente las actitudes y las concepciones educativas de los docentes ante la existencia y pujanza de la diversidad cultural en la escuela, y si la presencia de alumnos de culturas minoritarias constituyen un elemento significativo para el establecimiento de procesos de reflexión pedagógica y de incorporación de nuevas prácticas educativas en su quehacer cotidiano.

Así pues, resulta fundamental hacernos las siguientes preguntas: ¿Qué ideas pedagógicas podemos obtener de estos estudios? ¿Qué percepciones tienen los docentes sobre la diversidad cultural? ¿Cuáles son las concepciones educativas que tienen los profesores que trabajan en contextos educativos de diversidad cultural? Pues bien, la primera respuesta que podemos ofrecer es sencilla: el profesorado tiene una perspectiva diversa sobre la diversidad cultural. Esto implica que existen visiones o enfoques pedagógicos distintos que, en su traducción en la práctica se pueden distinguir de una manera más o menos definitoria. En este punto, en un trabajo anterior (Leiva, 2008), descubrimos la existencia de cuatro "miradas" sobre educación intercultural desde el punto de vista del profesorado: técnica-reduccionista, romántica-folclórica, crítica-emocional y reflexiva-humanista.

La perspectiva técnica-reduccionista es aquella que plantean los docentes que consideran que la educación intercultural es, más que otra cosa, una educación que se imparte en contextos educativos donde hay muchos alumnos inmigrantes y especialmente dirigida a ellos y a sus familias. La diversidad cultural es vista como un problema, $y$ las acciones educativas interculturales se contemplan como una respuesta educativa a problemas fundamentalmente lingüísticos, conductuales y de índole de curricular con alumnado inmigrante con problemas de incorporación tardía o desfase curricular significativo. 
La perspectiva romántica-folclórica, especialmente predominante en muchos centros es aquella donde el profesorado percibe y siente la interculturalidad como una propuesta fundamentalmente utópica y cuyo reflejo real en la práctica escolar es exaltar el mero conocimiento cultural de determinados aspectos de las culturas de los alumnos inmigrantes de sus centros escolares a través de fiestas y jornadas escolares específicas. Es la típica perspectiva de iniciación a la educación intercultural cuyo riesgo o déficit radica precisamente en que sólo permanezca en la valoración y conocimiento del folclore (baile, música, ropa, gastronomía, etc.) en momentos puntuales de la vida escolar, sin ningún tipo de vinculación o imbricación curricular por parte del profesorado. Además, en esta perspectiva resulta curioso que muchos docentes alaban la necesidad de contar con diferentes entidades socioculturales para la realización de actividades puntuales de carácter lúdico o formativo, pero no existe un claro compromiso por parte del docente en su colaboración o coordinación.

El enfoque o mirada crítica-emocional en educación intercultural se asocia a un profesorado comprometido con la diversidad cultural como un elemento de motivación educativa para la transformación, el cambio y la innovación curricular. La interculturalidad no se plantea como una propuesta dirigida a toda la comunidad educativa, y se trasciende el marco lectivo y de clase para dinamizar la escuela como un espacio donde tengan voz familias y alumnos autóctonos e inmigrantes para aprender a convivir juntos, y donde el contagio emocional del intercambio cultural es un elemento clave para la promoción positiva de la autoestima de los jóvenes inmigrantes, y, también, de la crítica constructiva de las diferentes identidades culturales grupales. En esta perspectiva lo importante es el fomento de la participación comunitaria y el empleo de todos los recursos educativos al alcance de la institución escolar, pero siempre con un compromiso crítico, ético y político del profesorado con las minorías étnicas y a favor de un curriculum contrahegemónico e intercultural.

El último enfoque o perspectiva intercultural sería la que denominamos humanista o reflexiva. Esta perspectiva también tiene el apoyo de un nutrido grupo de docentes que considera que la interculturalidad es un cambio actitudinal y de educación en valores. En esta perspectiva se defiende la importancia de manejar competencias interculturales en una comunicación que debe ser auténtica y empática por parte del docente hacia el alumnado y las familias inmigrantes, pero abriendo la necesidad de que educación intercultural sea un ejercicio de compartir significados culturales, donde lo importante no es tanto las actuaciones prácticas sino el sentido y sensibilidad que subyace en dichas prácticas (abiertas, colaborativas,...).

Sin ánimo de ser excesivamente exhaustivo en el análisis de estos modelos, sí es importante destacar que el estudio del pensamiento pedagógico intercultural del profesorado implica conocer las percepciones educativas en sus diferentes dimensiones: cognitiva, procedimental, ética y emocional. Así pues, mientras en el enfoque técnico-reduccionista la interculturalidad tiene un fuerte componente de acción educativa de carácter compensatorio, la perspectiva crítica defendería la vertiente crítica de la interculturalidad como herramienta dirigida a transformar el curriculum escolar hegemónico en un curriculum intercultural, que sea útil $y$ funcional para todos los alumnos, donde la diferencia cultural sea un valor educativo en la planificación e intervención didáctica (Sleeter, 2005). Desde este enfoque es obvia la consideración de que la interculturalidad es una respuesta educativa dirigida a todo el alumnado, esto es, no es una educación específica ni especial para el alumnado de origen inmigrante, sino que debe encararse desde la colaboración y participación crítica de todos los miembros de la comunidad educativa. En este punto, la posición reflexiva-humanista es similar a la crítica, y la única diferencia que podamos expresar se sitúa en el plano de la comunicación y de la innovación curricular. En el caso de la postura romántica-folclórica, los docentes perciben la interculturalidad como una respuesta educativa dirigida a toda la población escolar, pero haciendo especial hincapié en la dimensión cognitiva de la cultura.

En síntesis, podemos afirmar que, a pesar de que existe un desfase entre la teoría y la práctica de la interculturalidad, y aunque persista cierta idea de compensación educativa en el concepto de educación intercultural, son cada vez más los profesores que optan por la interculturalidad como alternativa pedagógica para construir una escuela más optimista y solidaria. La interculturalidad no tiene por qué centrarse exclusivamente en conocer al otro, sino en 
legitimar esa diferencia cultural y aprovecharla educativamente para enriquecer la convivencia y el aprendizaje cooperativo. Desde nuestro punto de vista, las actividades interculturales son realmente interculturales en la medida en que responden a un intento consciente y planificado de aprender a convivir en la diversidad como algo absolutamente ineludible.

“...nosotros hemos cogido una opción que es la interculturalidad, porque la multiculturalidad es solamente hacer cuentos, banderas.., y teníamos bastante..., mira, sinceramente, nosotros queremos quedarnos ahí por opción, es decir, creemos la interculturalidad como tú me das y yo te doy..., y es mucho lo que yo puedo aprender de tu cultura, y es mucho lo que puedes aprender de la mía..., y ahí está la riqueza, porque vamos en realidad a una sociedad plural, en la cual, tenemos que ver todo lo que podemos compartir..." (Fragmento de Entrevista a Directora de CEIP).

Por tanto, es fundamental plantear la interculturalidad desde su desarrollo práctico en los tres agentes claves de la comunidad educativa, es decir, profesorado, alumnado y familias. La educación intercultural implica además el conocimiento de la realidad de los países de origen de este alumnado y su acercamiento a las aulas españolas, la apuesta por el mantenimiento de la lengua y cultura de origen del nuevo alumnado y la apertura del centro escolar a formas distintas de ver, mirar, sentir y comprender la realidad social y educativa.

Es necesaria una mayor y mejor participación familiar, una formación intercultural reflexiva y crítica de los docentes, una optimización del uso de los recursos y apoyos que plantean las entidades sociales a los centros escolares, y, no menos importante, un apoyo decidido y contundente por parte de las administraciones educativas a aportar más recursos didácticos, materiales y humanos para que las escuelas puedan desarrollar en la práctica los principios y objetivos de la educación intercultural (García y Goenechea, 2009).

Principios y objetivos que deben ser construidos a partir de nuevas formas de participación escolar, con proyectos de innovación curricular y con apoyo decidido a los grupos de profesores que crean materiales educativos interculturales así como proyectos críticos de interculturalidad en su contexto escolar.

El éxito de la inclusión del alumnado inmigrante en los centros educativos de nuestro país depende en buena medida de las actitudes y los comportamientos que se desarrollen en el aula $y$ en el centro escolar en su conjunto por parte de alumnado, familias y profesorado, y en este caso, el papel del profesorado como agentes dinamizadores de la educación intercultural es trascendental. Ya ha pasado la época de la moda intercultural, y ya hoy las escuelas ofrecen recursos más o menos eficaces de compensación educativa y de apoyo a las dificultades lingüísticas en el caso de los alumnos inmigrantes no hispanoparlantes.

El reto de la inclusión del alumnado inmigrante pertenecía y pertenece al ámbito de la atención a la diversidad cultural del alumnado, pero la educación intercultural va más allá, tiene que ver con la construcción de la convivencia y la promoción de la participación y la innovación curricular y comunitaria. Hay, por tanto, una evolución conceptual y procedimental que se observa con nitidez al analizar el pensamiento pedagógico y la propia práctica educativa intercultural del profesorado.

Así pues, si tendríamos que decantarnos por defender una opción plantearíamos la necesidad de obviar dogmatismos y discursos retóricos, y sí seríamos comprensivos con las múltiples responsabilidades y funciones que tienen los docentes del siglo XXI. Esto no implica la renuncia a una perspectiva de indagación crítica en la interculturalidad, sino aceptar y comprender que la educación intercultural debe avanzar por los caminos trazados por equipos creativos de docentes preocupados y ocupados en aprovechar la diversidad cultural como un factor prioritario del curriculum y de la práctica docente, partiendo siempre de pequeñas acciones que vayan afianzando nuevas estrategias e innovaciones didácticas. Lógicamente esto supone abrirse a una perspectiva inclusiva y holística que aglutine lo positivo de las diferentes miradas educativas interculturales de los docentes con el objetivo de ir construyendo la interculturalidad en la escuela de manera eminentemente práctica, sin caer en activismos ni en posicionamientos idealizados, $\mathrm{y}$ sí en acciones educativas que fomenten el intercambio, la participación, el aprendizaje compartido, la innovación curricular y, sobre todo, la mejora de la convivencia escolar. 


\section{3.- LA CONVIVENCIA INTERCULTURAL COMO OBJETIVO DE LA ESCUELA INCLUSIVA.}

Si al analizar los discursos que existe entre el profesorado ante la presencia de alumnos inmigrantes en el sistema educativo, $y$ en definitiva, ante la inmigración, se constata la gran diversidad de opiniones y percepciones al respecto, no ocurre menos cuando se indaga en los discursos procedentes de las familias, tanto inmigrantes como autóctonas (Leiva, 2010). Por una parte, aparecen aquellos relatos que muestran el claro respeto hacia la diversidad cultural y que además consideran que es positivo y enriquecedor para sus hijos las relaciones interculturales. Y, por otra parte, también hay familias reticentes al intercambio cultural, no tanto por las connotaciones específicas de índole cultural o identitaria, sino sobre todo por las posibles repercusiones que dichas connotaciones pueden influir en el rendimiento académico de sus hijos y el nivel educativo de los centros escolares.

Según Sabariego (2002) la educación es una construcción social y dinámica que no es responsabilidad únicamente de la escuela ni tampoco de la familia; es un proceso en el que, conjuntamente, y de manera interdependiente, escuela, familia y sociedad aparecen como ejes relevantes e imprescindibles en el mismo. En torno a esta idea, que se refiere a las responsabilidades de los diferentes agentes en los procesos educativos, es necesario prestar atención a la participación de padres y madres en las escuelas interculturales e inclusivas.

Es cierto que la falta de participación familiar, el escaso protagonismo y compromiso de algunos padres y madres de alumnos en la educación de sus hijos se muestra como una de las principales preocupaciones por parte del colectivo de profesores en la actualidad, y es que se confirma la tendencia general y creciente de que los padres tienden a delegar exclusivamente la educación de sus hijos en la escuela, lo que constituye una dejación o desinterés de su responsabilidad familiar en la tarea educadora.

Ante ello, el docente es la persona cuya sensibilidad y compromiso con una educación intercultural auténtica puede ayudar, no sólo al alumno sino también a la propia familia, conociendo y respetando su idiosincrasia cultural y su propia identidad, haciéndoles ver la importancia de establecer vínculos comunicativos de encuentro e interacción cultural, donde su participación y e implicación en las acciones desarrolladas en la escuela sean vistas como necesarias y enriquecedoras para la propia salud vital de un clima escolar positivo.

Tal y como subraya Soriano (2009), el protagonismo del docente en las escuelas cuya diversidad la definen, es de absoluta trascendencia sobre todo cuando el fenómeno de la diversidad cultural es vista como algo perturbador o negativo, o incluso algo ajeno o indiferente en el contexto social.

La práctica educativa intercultural viene determinada por dos aspectos fundamentales. En primer lugar, la concepción pedagógica que tienen los docentes sobre el significado de la interculturalidad, y en segundo lugar, la formación intercultural del profesorado. Estos dos aspectos son básicos para entender que, en la actualidad, la práctica de la educación intercultural es muy diversa y heterogénea, y que la traducción de los principios y valores de la educación intercultural modula generalmente entre el deseo y la realidad, esto es, entre el deseo de ser una propuesta pedagógica crítica, y la realidad de ser una prolongación de un tipo específico de educación compensatoria.

Partimos de la idea que construir interculturalidad precisa de la necesaria posibilidad de afirmar la propia cultura en su relación con las otras culturas. Y que esta afirmación se realice mediante un proceso donde todos y todas podemos aportar, y donde todas estas aportaciones sean sujeto de intercambio y de valoración crítica (Aguado, 2003). Es así como, desde la expresión de la diversidad, la relación intercultural puede construirse en un medio de cohesión e integración social. Y, lo más difícil, el establecimiento de un marco de relaciones donde se facilita la interacción cultural en condiciones de igualdad de oportunidades.

El fin de la educación intercultural es lograr la convivencia, el respeto y la valoración mutua entre los alumnos para que ese clima de entendimiento y de tolerancia se traslade a la sociedad en la que vivimos. A pesar de ello, sabemos que uno de los problemas que repetidamente se manifiesta a la hora de establecer relaciones y vínculos interculturales ha sido la relevancia que hemos otorgado a las diferencias en detrimento de los aspectos que nos unen, que nos igualan, y en la base de dichos aspectos hay uno fundamental: todos somos 
personas que tenemos múltiples identidades personales. Precisamente ese debe ser el fundamento de la educación intercultural, es decir, para que ese deseo de respeto y de entendimiento pueda transformarse en una realidad será necesario que todos los alumnos, que todas las personas se sientan valoradas, apreciadas y aceptadas por quiénes son y no únicamente por su procedencia y cultura de pertenencia.

Por todo ello, el centro educativo intercultural, la escuela intercultural, debe ser un espacio privilegiado de vida cultura y comunitaria. Es decir, los colegios e institutos de un barrio deben constituirse en centros generadores de participación comunitaria, ser permeables y estar abiertos a conformar en ellos una cultura escolar diversa, compleja y dinámica. La escuela intercultural e inclusiva debe apostar claramente por la transformación crítica de la realidad que le ha tocado vivir, y debe servir como canal de diversas expresiones éticas y culturales. En este punto, podemos afirmar sin miedo a equivocarnos que el siguiente paso de la interculturalidad debe ser la generación en la escuela de un debate en torno a la cultura de la diversidad. Es decir, se debe pasar de los procesos de enfatización positiva de la diferencia cultural a la valoración global de una cultura de la diversidad que, en sí misma, sea posibilitadora de una escuela más democrática, equitativa, solidaria y crítica (López Melero, 2004). La escuela no sólo debe generar conocimiento significativo, también ciudadanía crítica e intercultural, de ahí la importancia de que los centros educativos superen reduccionismos y se abran sin temor a su apertura a la comunidad.

Los centros escolares deben superar la celebración de las festividades extraacadémicas desde posiciones exclusivistas y de puertas para adentro. Más bien al contrario, las instituciones educativas deben implicarse en la generación de comisiones y diversas instancias participativas ampliamente representativas de la comunidad educativa, donde participen madres y padres, inmigrantes y autóctonos, asociaciones y entidades sociales, clubes juveniles, etc. El objetivo debe plantearse en términos de comunidad, de una educación intercultural dirigida a promover la participación comunitaria en la escuela, de tal manera que se negocie, se reconstruya $y$ se gestione eficazmente las diversas actuaciones escolares (formales y no formales) para que la escuela sea un espacio de encuentro en torno a las acciones educativas interculturales compartidas y comunitarias de todos y para todos.

En este marco de reflexión pedagógica, inscribimos algunas reflexiones de docentes que tienen una visión crítica de la interculturalidad. Una visión que va más allá de la educación compensatoria y de la educación para la paz. En efecto, la educación intercultural se postula como un enfoque pedagógico transformador al considerar la educación como una construcción eminentemente social y comunitaria. Por esta razón, este posicionamiento inclusivo es el que apuesta para que el docente participe activamente no solamente en el plano meramente escolar, sino también en el plano de la participación y la vida política local. En verdad, no es descabellado plantear que el profesorado, como primer agente social que recibe el impacto de la inmigración en la escuela, tenga una voz privilegiada que deba ser oída en el diseño de políticas públicas (socioeducativas) sobre cómo afrontar la inclusión social y educativa de los alumnos y las familias inmigrantes en la comunidad.

Por otro lado, Santos Rego (2009) y Montón (2004) ya apuntan a cómo la participación de las organizaciones y entidades sociales juegan un papel cada día más relevante, sobre todo en los contextos escolares de diversidad cultural, que en términos generales, son más susceptibles de recibir apoyos y ayudas de diversa índole por parte de estas instituciones (ONGs, AA.VV.s...). El problema es que el profesorado se mueve a menudo en un sistema de comunicación cerrado debido, en ocasiones, a la inseguridad que le suscita la falta de dominio de algunos temas de actualización pedagógica (Irvine, 2003).

Así mismo, hay algunos profesores que no aprovechan el potencial didáctico que le ofrecen muchas ONGs y grupos sociales que podrían colaborar en la transformación intercultural del curriculum escolar. En efecto, a pesar de que es cierto que cada día acuden con más frecuencia estas entidades a trabajar a la escuela, todavía persiste la idea de que la institución escolar no es el espacio natural para estas entidades sociales, las cuales acostumbran a basar su estrategia de acceso al centro escolar mediante la elaboración de materiales didácticos externos o bien la realización de algún tipo de actividad puntual generalmente lúdica- en el contexto educativo formal. En todo caso, sí tenemos que confirmar que estas entidades no sólo trabajan en horario extraescolar, sino que ya actúan en el horario 
escolar, dotando de una gran vitalidad y participación a la institución educativa. El reto está en consolidar este trabajo a través de dos estrategias metodológicas de enorme potencialidad educativo: la acción tutorial del profesorado y el aprendizaje servicio. En ambos elementos es necesaria la formación del profesorado en competencias interculturales y se requiere un trabajo formativo de carácter interdisciplinar entre profesores, familias, mediadores interculturales, educadores sociales y trabajadores sociales.

Es cierto que la primera estrategia es básica y se viene desarrollando entendiendo la interculturalidad como un constructo más en la educación en valores o como tema transversal. No obstante, aparece con fuerza la necesidad de imbricar la interculturalidad dentro del curriculum escolar y también implicando acciones educativas que conecten la educación con el servicio a la comunidad, esto es, que se diseñen y desarrollen propuestas que el alumnado pueda desarrollar en sus contextos más cercanos (familia, barrio, asociaciones de vecinos, etc.) para la promoción de la interculturalidad y la cultura de la diversidad (p.e. realizar estudios sobre la inmigración en el barrio, analizar la integración del colectivo inmigrante, hacer juegos cooperativos de paz y diversidad, estudiar conflictos interculturales, etc.)

\section{4.- LA EDUCACIÓN INTERCULTURAL EN UNA ENCRUCIJADA DE CAMINOS: DE LA TEORÍA A LA PRÁCTICA.}

Tal y como ponen de manifiesto diferentes estudios e investigaciones (Aguado, 2003; Jordán, 1999 y 2007; Soriano, 2004 y 2008), la interculturalidad es concebida como una propuesta educativa reflexiva de enorme interés y potencialidad para los docentes, pero su traducción en la práctica escolar está llena de contradicciones y ambigüedades, que nos hacen repensar la interculturalidad desde diferentes enfoques -y significados- para comprender el pensamiento pedagógico que el profesorado tiene acerca de esta propuesta de acción educativa. Sin embargo, el profesorado de nuestro país es consciente de la necesidad que existe de construir escuelas que sean verdaderos espacios de diálogo intercultural, y donde la cultura de la diversidad sea un pilar básico de una educación que forme a ciudadanas y ciudadanos democráticos y respetuosos en la diversidad social y cultural. A raíz de la importancia creciente entre los conceptos de interculturalidad, diversidad cultural y convivencia, decidimos realizar un estudio sobre el pensamiento y la práctica pedagógica intercultural del profesorado.

\section{Método.}

En este estudio se ha tenido en cuenta la necesidad de apostar por una metodología cualitativa (mediante estudios de casos en profundidad y entrevistas en profundidad) ya que nos podía revelar importantes sinergias y posibilidades de indagación y conocimiento intercultural. Por un lado, se han desarrollado cuatro estudios de casos en profundidad, donde hemos estudiado las concepciones pedagógicas que sobre la interculturalidad y la convivencia tienen cuatro docentes que pertenecen a distintos centros educativos de educación infantil y primaria de la provincia de Málaga, y que realizan distintas funciones docentes u ocupan diferentes puestos 0 responsabilidades educativas.

\section{Instrumento.}

Estos estudios cualitativos han empleado como instrumentos de recogida de información la entrevista, la recopilación documental y el diario investigador. Por otro lado, hemos realizado cuarenta y cuatro entrevistas en seis centros educativos más de la provincia de Málaga, tres institutos de educación secundaria y tres colegios de educación infantil y primaria.

\section{Muestra.}

La muestra que hemos empleado en la realización de estas entrevistas está conformada por un total de cuarenta y cuatro personas, entre profesorado, alumnado y familia. La muestra fue elegida teniendo en cuenta su nivel de representatividad, es decir, son personas (profesorado, alumnado, familia) que están insertas en una realidad educativa de diversidad cultural, lo cual constituyó un criterio fundamental en la selección de los informantes.

\section{Análisis de datos.}

Cabe destacar que para el análisis de todos los datos cualitativos recogidos en este estudio utilizamos el programa informático Nudist-Vivo 2.0. A partir de la introducción de todas las informaciones en el formato requerido por este software (entrevistas en profundidad, diario investigador, documentos de los centros educativos, observaciones), hicimos una codificación informática o categorización segmentada de textos en base a unidades 
temáticas. Exponemos ahora algunas consideraciones sobre ello a partir de las conclusiones del mencionado estudio (Leiva, 2007).

\section{Resultados.}

En primer lugar, el profesorado se encuentra comprometido con que los principios de la interculturalidad impregnen y formen parte necesariamente de los principios educativos de sus escuelas, y que las actividades interculturales son fundamentales para favorecer la integración efectiva del alumnado inmigrante.

“(...) pero sí es verdad que hay que seguir avanzando, precisamente en el objeto de la interculturalidad.., en el Inter, porque yo quiero que las madres inmigrantes participen en la vida del colegio, es fundamental para el colegio.., y creo que hay que aceptar lo bueno de todas las culturas, quiero que seamos capaces de integrarlos sin perder su identidad, que no hay que perderla, de ninguna de las maneras..., así, todas las culturas, y esto claro, esto puede producir en un colegio como el nuestro, una riqueza inmensa..." (Fragmento de Entrevista a Profesora de $6^{\circ}$ de Primaria, Ep.C4).

En segundo lugar, podemos apreciar un cambio muy significativo e interesante en relación al concepto de interculturalidad, y es que el profesorado se expresa positivamente al entender que las actividades interculturales tienen que dirigirse a toda la población escolar, esto es, no solamente al alumnado inmigrante sino a todo el alumnado sin ningún tipo de exclusión. Esto, sin lugar a dudas, es una idea clave que podemos extraer de los datos (tanto cualitativos como cuantitativos) en nuestro estudio, y que nos ofrece una orientación manifiestamente favorable a considerar la interculturalidad desde una dimensión más abierta, optimista y comprometida con toda la comunidad educativa.

Existe un alto grado de compromiso por parte de los docentes en desarrollar acciones educativas interculturales. De hecho, el perfil del profesorado que trabaja en las escuelas interculturales es cada vez más el de un profesorado implicado y concienciado en desarrollar ideas pedagógicas innovadoras y transformadoras ${ }^{1}$. En este sentido, estos docentes consideran que los alumnos/as inmigrantes necesitan sentir "valorada su cultura (...), que el curriculum que se diseñe en cualquier tipo de centro tiene que responder a la diversidad cultural, y la diversidad cultural significa responder a los valores culturales y a las características de cada uno de ellos" (Fragmento de Entrevista a Profesor de $5^{\circ}$ de Primaria, Ep.C1).

En tercer lugar, otra conclusión que se desprende de nuestro estudio es que el profesorado concibe la educación intercultural en términos de reflexión profunda sobre la educación actual, es decir, es una opción pedagógica que emerge con fuerza a partir de la reflexión sobre la actual situación de creciente diversidad cultural en las escuelas. Sin embargo, hay que decir que son muchos los profesores que opinan que la interculturalidad no solamente puede quedarse en el aspecto reflexivo, sino que también tiene que dar una respuesta práctica y ajustada a la realidad educativa compleja que se vive en las escuelas de diversidad. Ahora bien, es cierto que todavía son pocos los docentes que contemplan la interculturalidad como una propuesta crítica y transformadora en educación. Sin embargo, existe una creciente tendencia a concebir la interculturalidad no solo en términos de conocimiento cultural (dimensión cognitiva), sino que los aspectos afectivos (dimensión emocional) también sean considerados como claves fundamentales para llevar a la práctica una auténtica educación intercultural.

\footnotetext{
${ }^{1}$ Esto es un aspecto que ha sido confirmado en los estudios cualitativos de casos de dicho trabajo de investigación (Leiva, 2007), y es que, no sólo el profesorado en comisión de servicios y adscrito a proyectos de educación intercultural, está claramente comprometido con el desarrollo de la interculturalidad. Estos docentes, así como el profesorado de aula ATAL, están dinamizando al resto de profesores y profesoras de sus respectivos centros, lo que está favoreciendo su actitud positiva hacia la educación intercultural. Además, estos colegios están elaborando propuestas prácticas que han venido a estimular en la provincia de Málaga el interés por este tipo de proyectos pedagógicos. Ejemplo de ello es la publicación del libro del Dr. Julio Vera, titulado Propuestas y experiencias de Educación Intercultural (2008), publicado por la Fundación Santa María (Madrid), donde se recogen algunas experiencias, actividades y materiales interculturales que se desarrollan actualmente en estos centros educativos (Ver pp.151-165).
} 
“...es empatía, ponerme en la piel..., acercarme a ellos. $Y$ ellos te ven que les entiendes, pero sobre todo es fundamental respetarles, quererles. Nunca nadie puede humillar a un niño, pero nunca...; tú tienes que acercarte a ellos, darles afecto. Además tú tienes relación con su casa, conoces a sus padres..." (Entrevista a Directora de colegio de infantil y primaria, Ep.C2).

En cuarto lugar, hay que señalar que diferentes agentes de la comunidad educativa valoran muy positivamente la diversidad cultural que implica la acogida de alumnado inmigrante en sus centros educativos, y esto es confirmado por una orientadora que afirma que "los niños de otras culturas son una gran riqueza (...), y son buenos alumnos, la mayoría, son niños más centrados, (y) no tienen ningún tipo de conflictos violentos"2. Sin lugar a dudas, ésta es una idea relevante en nuestro estudio, ya que se confirma la aceptación y valoración positiva de las diferencias culturales por parte de los docentes de las escuelas interculturales.

Igualmente, nos indica una tendencia que hemos podido confirmar en nuestro estudio y en otros trabajos recientes de investigación (Merino, 2003; Soriano, 2008), y es la idea cada vez más extendida entre el profesorado de que el alumnado inmigrante es un buen alumnado en términos generales, con predisposición al estudio y a un comportamiento correcto y respetuoso con las normas de convivencia en nuestras instituciones escolares. Esto ha sido confirmado en distintos estudios realizados en la provincia de Málaga, y no se refieren especialmente al alumnado inmigrante de la Costa (p.e., ingleses, franceses o alemanes), sino fundamentalmente a alumnos y alumnas de origen magrebí y de origen latinoamericano (Ruiz, 2005; Rascón, 2006).

La cultura de la diversidad como fundamento de la interculturalidad tiene importantes implicaciones pedagógicas que afectan e influyen a todos los aspectos de la educación. No obstante, en este artículo vamos a centrarnos en las implicaciones pedagógicas que se derivan de su análisis crítico en relación a la necesaria formación intercultural de los profesores. Dicho esto, tenemos que subrayar que

\footnotetext{
${ }^{2}$ Fragmento de Entrevista a Orientadora de Equipo de Orientación Educativa de Zona CAEP (Centros de actuación educativa preferente), Eo, C1.
}

el profesor competente, desde la perspectiva intercultural, es aquel que tiene la habilidad de interactuar con “otros” (alumnos/as y familias inmigrantes), de aceptar otras perspectivas y percepciones del mundo, de mediar entre diferentes perspectivas y de ser consciente crítica y reflexivamente de sus propias valoraciones sobre la diversidad cultural. La competencia intercultural se compone de conocimientos, habilidades y actitudes de respeto y aceptación de la diversidad cultural como un valor educativo de primer orden en el quehacer pedagógico. Las actitudes (de apertura, voluntad de relativizar las propias creencias y comportamientos, de empatía...) constituyen la base de la competencia intercultural del profesorado.

"...hay algunos que se dan cuenta y cambian un poquito el chip, otros se acoplan sin estar convencidos, hay de todo..., y verdaderamente todas las estrategias pasan por todo un proceso de comprensión, de trabajo de competencias, de habilidades para que esos conflictos realmente se solucionen..., y claro, todo esto que te estoy hablando de comprender, de saber, de que tú no puedes tratar a un niño de mala manera..., tienes que tener un trabajo previo, tú cuando a un alumno lo coges ya en el conflicto, ya has perdido la pelea, tu tienes que detectar antes que van a surgir conflicto, entonces, poner antes el parche antes de que salga el grano..., ir evitando, ir previniendo, ir haciendo trabajo sobre eso..." (Fragmento de Entrevista a Profesor de $4^{\circ}$ Educación Primaria, Ep.C2).

Aunque es verdad que no todo el profesorado de las escuelas interculturales está verdaderamente implicado en el reconocimiento de la diversidad cultural como un capital educativo de primer orden en su práctica educativa, es cierto que a nivel conceptual y de pensamiento pedagógico del profesorado, la diversidad cultural está siendo progresivamente considerada como un factor positivo para promover una educación de calidad en sus centros educativos.

"No, al revés; yo, cada vez que he hablado con ellos, los he visto muy receptivos, y en ningún momento. Vamos, yo siempre lo pongo como ejemplo, porque no es muy normal, que todo el mundo sea receptivo." (Fragmento de 
Entrevista a Profesora de Aula Temporal de Adaptación Lingüística, Ep.C4)

En efecto, las competencias interculturales no solamente tienen que ver con el simple hecho de aceptar y reconocer la importancia de la diversidad cultural, también tienen una dimensión afectiva de valores de cercanía y respeto hacia el alumno inmigrante $\mathrm{y}$ sus familias, como elemento clave en el establecimiento de relaciones de confianza para que se promueva un clima de respeto e igualdad en el centro educativo.

“...ellos ven que tú muestras cercanía y respeto..., así como si tú te paras en la calle y hablas con ellos.., porque de alguna manera el prototipo que existe de directora es que es una persona que parece que está sobre un podium, o que está por encima..., entonces, claro, a mí ese tema no me sirve, no soy así, así que yo, desde que entro en el colegio, yo sé que mi trabajo es servir a los demás, pero sobre todo soy una persona. $Y$ desde que entro al colegio me voy encontrando a madres, gitanas, judías, musulmanas.., y de todos los colores, y me voy parando con todos, oyendo sus problemas, y queriendo escuchar las cosas que me quieren contar..." (Fragmento de Entrevista a Directora de Educación Primaria, Ep.C2).

Dicho esto, nos gustaría apuntar ahora algunas propuestas pedagógicas derivadas precisamente de la necesidad de mejorar la comunicación y la competencia intercultural del profesorado, atendiendo a la necesidad de generar procesos de reflexión sobre la cultura de la diversidad en la escuela:

a) Potenciar la autoestima de los alumnos/as inmigrantes, ayudándoles a desarrollar la confianza en su habilidad para progresar en sus relaciones sociales y emocionales con sus compañeros de clase y el profesorado.

b) Facilitar a todo el alumnado de las escuelas interculturales el desarrollo y puesta en práctica de habilidades de conocimiento y respeto a la diversidad cultural existente en sus centros educativos.

c) Favorecer la interacción y la comprensión cultural y social de todos los alumnos/as y sus familias, a través del desarrollo de una perspectiva amplia de edu- cación en valores democráticos en el marco de la sociedad en la que viven.

d) Proporcionar al alumnado inmigrante ayuda y comprensión para desarrollar el conocimiento, las actitudes y las habilidades necesarias para mantener, siendo conscientes críticamente de su identidad individual y cultural, sus raíces culturales sin perder de vista la importancia de su integración plena en la sociedad de acogida, a través del aprendizaje crítico de referentes éticos, sociales y emocionales que le permitan mejorar sus expectativas educativas y sociales.

A la luz de estas propuestas, nos parece necesario indagar en un modelo educativo intercultural e inclusivo que englobe los aspectos positivos de los distintos enfoques que hemos estudiado en el presente trabajo. En este sentido, estamos de acuerdo con Aguado (2003) y Soriano (2008), en la necesidad de ir construyendo un modelo inclusivo de acción educativa para las escuelas interculturales, y en términos generales, para todas las escuelas sin ningún tipo de excepciones. Entre las principales características que pueden definir este modelo, proponemos las siguientes:

- El profesorado debe promover actitudes democráticas y solidarias.

- La comunidad educativa necesita reflejar y legitimar la diversidad cultural, lingüística y étnica existente en su contexto socioeducativo.

- El curriculum y los materiales didácticos deben presentarse desde diferentes perspectivas culturales, sobre conceptos, procedimientos y valores.

- Tanto el profesorado como el alumnado necesitan adquirir competencias interculturales para la mejora de la convivencia y la gestión positiva de los conflictos interculturales.

- Los equipos directivos pueden y deben impulsar la participación activa de todas las familias y agentes educativos del contexto escolar.

- La comunidad educativa requiere de todos los apoyos sociales y escolares disponibles para aprovechar educativamente la diversidad cultural como una riqueza y no como un problema.

- El docente debe convertirse en creador y 
recreador de materiales curriculares interculturales, formándose en estrategias educativas interculturales inclusivas y creativas, así como en destrezas socioemocionales de afrontamiento de los conflictos interculturales.

\section{5.- ALGUNAS CONCLUSIONES PARA LA REFLEXIÓN EDUCATIVA.}

Nuestra propuesta va en la línea de construir una educación intercultural inclusiva donde el proyecto educativo de centro (PEC) se haga no desde una perspectiva técnica o administrativa, sino fundamentalmente crítica y transformadora, con la participación e implicación de alumnos, familias y entidades sociales en el marco de la comunidad educativa. Se trataría de naturalizar la presencia y la relación educativa del profesorado con el resto de agentes de la comunidad educativa desde un enfoque de simetría participativa, es decir, que estas entidades y organizaciones sociales del barrio puedan concretar su representación y acción en la escuela a través de una comisión o un consejo que permita la actualización curricular permanente.

También, resulta ineludible afirmar que la formación del profesorado en educación intercultural es básica para llevar a buen término los objetivos propuestos por la misma. Esta formación no sólo puede ir exclusivamente dirigida al colectivo docente sino para toda la comunidad educativa, y es que todos somos piezas claves y singulares para avanzar hacia la interculturalidad.

Esta formación intercultural tiene que estar ajustada y contextualizada a la realidad profesional que los docentes viven cada día en sus aulas y escuelas. El profesorado de hoy no valora positivamente los conocimientos teóricos sobre interculturalidad de manera aislada, sino que estos conocimientos de formación intercultural deben estar vinculados y relacionados dentro de una metodología participativa donde juegue un papel clave la simulación y la formación práctica con el estudio de casos particulares de conflictos $\mathrm{o}$ de situaciones de interculturalidad en la convivencia.

$\mathrm{Y}$ es que, si pretendemos construir aulas $\mathrm{y}$ escuelas interculturales, porque creemos que es en sí mismo un propósito ineludible al que debe responder hoy en día la vida de todos los centros educativos, es imprescindible asumir la diversidad como algo positivo y enriquecedor para la propia convivencia social y educativa. Y es que, en definitiva, la diversidad es lo común y lo común es la diversidad, y aplicado a la educación, esto nos lleva a indagar más si cabe en la riqueza de la humanidad y en el necesario desarrollo de metodologías y acciones educativas inclusivas e interculturales.

\section{6.- REFERENCIAS BIBLIOGRÁFICAS.}

Aguado, T. (2003). Pedagogía intercultural. Madrid: McGraw-Hill Interamericana.

Banks, J.A. (2008). Diversity, Group Identity and Citizenship Education in a Global Age, Educational Researcher, 37(3), pp. 129-139.

Bartolomé, M. (2002). Identidad y ciudadanía: un reto a la educación intercultural. Madrid: Narcea.

C.E.J.A. (2010). La Educación en Andalucía, 20092010. Sevilla: Unidad Estadística de la Consejería de Educación de la Junta de Andalucía.

Essomba, M. A. (2006). Liderar escuelas interculturales e inclusivas. Equipos directivos y profesorado ante la diversidad cultural y la inmigración. Barcelona: Graó.

García, J. A. \& Goenechea, C. (2009). Educación intercultural. Análisis de la situación y propuestas de mejora. Madrid: Wolters Kluwer.

Irvine, J.J. (2003). Educating teachers for divesity: Seeing with a cultural eye. New York: Teachers College Press.

Jordán, J.A. (1999). El profesorado ante la educación intercultural. En M.A. ESSOMBA (Coord.) Construir la escuela intercultural. Reflexiones y propuestas para trabajar la diversidad étnica y cultural (pp. 65-73). Barcelona: Graó.

Jordán, J.A. (2007). Educar en la convivencia en contextos multiculturales. En E. SORIANO, Educación para la convivencia intercultural (pp.59-94). Madrid: La Muralla.

Leiva, J. (2007). Educación y conflicto en escuelas interculturales. Málaga: Spicum.

Leiva, J. (2008). La escuela como espacio privilegiado para el desarrollo de la educación intercultural: el compromiso de los profesores. Campo Abierto, 27(1), 13-35.

Leiva, J. (2010). Práctica de la interculturalidad desde la perspectiva docente: análisis y propuestas pedagógicas. Cultura y Educación, 22(1), 67-84.

López Melero, M. (2004). Construyendo una escuela sin exclusiones. Archidona (Málaga): Aljibe.

M.E.C. (2009). Datos y Cifras. Curso escolar 2009/2010. Informe sobre el estado y situación del Sistema Educativo. Madrid: Centro de Investigación y Documentación Educativa. 
Montón, Ma. J. (2004). La integración del alumnado inmigrante en el centro escolar. Barcelona: Graó.

Sabariego, M. (2002). La educación intercultural ante los retos del siglo XXI. Bilbao: Desclée de Brouwer.

Santos Rego, M.A. (2009) Políticas educativas y compromiso social. El progreso de la equidad y la calidad, Barcelona: Octaedro.
Sleeter, C.E. (2005). Un-standardizing curriculum: Multicultural teaching in the standards-based classroom. New Cork: Teachers College Press.

Soriano, E. (2008). Educar para la ciudadanía intercultural y democrática. Madrid: La Muralla.

Soriano, E. (2009). Vivir entre culturas: una nueva sociedad. Madrid: La Muralla.

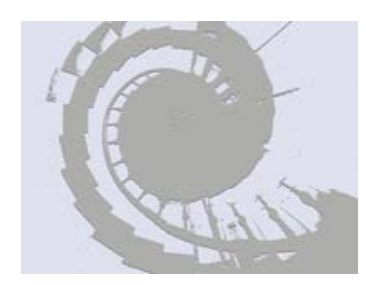

